\title{
KOMLÓS PROPERTIES IN BANACH LATTICES
}

\author{
E. Y. EMELYANOV ${ }^{1}$, N. ERKURŞUN-ÖZCAN ${ }^{2}$, S. G. GOROKHOVA ${ }^{3}$
}

\begin{abstract}
Several Komlós like properties in Banach lattices are investigated. We prove that $C(K)$ fails the oo-pre-Komlós property, assuming that the compact Hausdorff space $K$ has a nonempty separable open subset $U$ without isolated points such that every $u \in U$ has countable neighborhood base. We prove also that for any infinite dimensional Banach lattice $E$ there is an unbounded convex uo-pre-Komlós set $C \subseteq E_{+}$ which is not uo-Komlós.
\end{abstract}

\section{INTRODUCTION}

In recent paper [5], the unbounded order convergence in Banach lattices was deeply studied. Among other things, this development has lead to study of generalizations of the Komlós celebrated theorem [7] to the Banach lattice setting. The authors of [5] did their generalization through $A L$ representations of a Banach lattice with a strictly positive order continuous functional, replacing almost everywhere convergence by unbounded order convergence. Beside such a natural extension, many questions on generalized Komlós properties are still requiring an investigation.

In the present paper, we study Komlós properties in more breadth settings, than for the uo-convergence. In Section 2, we define and investigate several Komlós properties for different modes of boundedness and convergence in a Banach lattice. Section 3 is completely devoted to Komlós properties in Banach lattices of continuous functions. Section 4 is dealing with so-called Komlós sets.

As the nature of the Komlós theorem is sequential, we restrict ourselves to sequential convergences in Banach lattices. For unexplained terminology and notations we refer the reader to $[1,2,3,4,4,5,6$. In the present paper, $E$ stands for a real Banach lattice.

Date: 06.10 .2017 .

2010 Mathematics Subject Classification. 46B42.

Key words and phrases. Banach lattice, o-convergence, uo-convergence, unconvergence, Komlós properties, Komlós sets, space of continuous functions. 


\section{Komlós LiKe PRoperties in BANACH lattices}

Let $x_{n}$ be a sequence in $E$ and $x \in E$. Recall that:

(1) $x_{n}$ converges in order to $x$ (we write $x_{n} \stackrel{\circ}{\rightarrow} x$ ), if there is a sequence $y_{n}$ decreasing to 0 (we write $y_{n} \downarrow 0$ ) with $\left|x_{n}-x\right| \leq y_{n}$ for all $n$;

(2) if $x_{n}$ converges in norm to $x$, we write $x_{n} \stackrel{\mathrm{n}}{\rightarrow} x$;

(3) $x_{n}$ is unbounded order convergent to $x$ (we write $x_{n} \stackrel{\text { uo }}{\longrightarrow} x$ ) if $\mid x_{n}-$ $x \mid \wedge u \stackrel{\circ}{\rightarrow} 0$ for every $u \in E_{+} ;$

(4) if $\left|x_{n}-x\right| \wedge u \stackrel{\mathrm{n}}{\rightarrow} 0$ for every $u \in E_{+}$, we write $x_{n} \stackrel{\text { un }}{\longrightarrow} x$ and say that $x_{n}$ is unbounded norm convergent to $x$.

The main motivation for the present paper is the following classical result [7].

Theorem 1 (Komlós). Let $E=L_{1}(\mu)$, where $\mu$ is a probability measure. Then, for every norm bounded sequence $x_{n}$, there is a subsequence $x_{n_{k}}$ such that the Cesáro means $\frac{1}{m} \sum_{j=1}^{m} x_{n_{k_{j}}}$ of any further subsequence $x_{n_{k_{j}}}$ converge almost everywhere to some $x \in X$.

The Komlós theorem has initiated many investigations and was extended recently for Banach lattices [5] by replacing a.e.-convergence with uo-convergence. This development has inspired the following definition.

Definition 1. A Banach lattices $E$ is said to have ab-Komlós property (respectively, ab-pre-Komlós property) if, for every a-bounded sequence $x_{n}$ in $E$, there exist a subsequence $x_{n_{k}}$ and an element $x \in E$ such that

$$
x=b-\lim _{m \rightarrow \infty} \frac{1}{m} \sum_{j=1}^{m} x_{n_{k_{j}}}
$$

for any subsequence $x_{n_{k_{j}}}$ of $x_{n_{k}}$ (respectively, the sequence $\frac{1}{m} \sum_{j=1}^{m} x_{n_{k_{j}}}$ is bCauchy for any subsequence $x_{n_{k_{j}}}$ of $x_{n_{k}}$ ).

Here, a-boundedness stands for o- or $n$-boundedness; and b-convergence stands for $\mathrm{o}^{-}, \mathrm{uo-}, \mathrm{n-}$, or un-convergence.

Clearly, it suffices to check $a b$-Komlós and $a b$-pre-Komlós properties only for sequences in $E_{+}$. Furthermore, no-Komlós implies oo-Komlós and, if $E$ is $\sigma$-Dedekind complete, they coincide with no-pre-Komlós and oo-pre-Komlós properties respectively.

nuo-Komlós and nuo-pre-Komlós properties were introduced in [5, Def.5.1] under the names of Komlós and pre-Komlós properties respectively. The Komlós Theorem 1 has been extended further in [5, Prop.5.13] as follows. 
Proposition 1 (Gao-Troitsky-Xanthos). Let $E$ be a regular sublattice of an order continuous Banach lattice $F$. Then $E$ has the nuo-pre-Komlós property. Moreover, $E$ has the nuo-Komlós property iff it is sequentially boundedly uo-complete.

Let us mention also the next corollary (see [5, Cor.5.14]) of [5, Prop.5.13].

Corollary 1 (Gao-Troitsky-Xanthos). Every order continuous Banach lattice $E$ has the nuo-pre-Komlós property. Moreover, $E$ has the nuo-Komlós property iff it is a KB-space.

Proposition 2. Every o-continuous Banach lattice $E$ has the nun-preKomlós property. Moreover, if $E$ is a KB-space then $E$ has the nun-Komlós property.

Proof. It follows from Corollary 1 since uo-convergence implies un-convergence (see [4, Prop.2.5]).

Example 1. The Banach lattice c of real convergent sequences fails the oo-Komlós (and hence no-Komlós) property. To see this, take the sequence $x_{n}=\sum_{j=1}^{n} e_{2 j}$ in $[0, \mathbb{1}] \subset c$. Clearly, for any subsequence $x_{n_{k_{j}}}$, the sequence $\frac{1}{m} \sum_{j=1}^{m} x_{n_{k_{j}}}$ is uo-divergent, and also o-divergent, since it is order bounded. It shows also that $c$ fails the nuo-Komlos property. Since the sequence $\frac{1}{m} \sum_{j=1}^{m} x_{n_{k_{j}}}$ is not n-Cauchy, c fails the nn-pre-Komlós property as well.

It can be easily seen that c has oo-, ouo-, and nuo-pre-Komlós property (cf. also [5, Cor.5.10]).

Proposition 3. Any o-continuous Banach lattice E has oo- and on-Komlós property.

Proof. Let $x_{n} \in[-u, u]$ for all $n$. By [5, Cor.5.14], $E$ has nuo-pre-Komlós property, and hence ouo-pre-Komlós property. So, there exists a subsequence $x_{n_{k}}$ such that any sequence $\frac{1}{m} \sum_{j=1}^{m} x_{n_{k_{j}}} \subset[-u, u]$ is uo-Cauchy, and hence $o$-Cauchy. By o-continuity of the norm, $E$ is Dedekind complete. It follows that there exist $y \in E$ with $y=o-\lim _{m \rightarrow \infty} \frac{1}{m} \sum_{j=1}^{m} x_{n_{k_{j}}}$ for any subsequence $x_{n_{k_{j}}}$ of $x_{n_{k}}$. Using o-continuity once more, we get $y=n-\lim _{m \rightarrow \infty} \frac{1}{m} \sum_{j=1}^{m} x_{n_{k_{j}}}$. 


\section{Komlós properties in BAnach lattices of CONTINUOUS FUNCTIONS}

Notice that, in $E=C(K)$ : oo-Komlós, no-Komlós, ouo-Komlós, and nuoKomlós properties coincide. The same is true for oo-, no-, ouo-, and nuopre-Komlós properties. Furthermore, $n n$-Komlós property coincides with both $n n$-pre-Komlós and on-pre-Komlós properties.

In view of Example 1, the Banach lattice $c \cong C(\mathbb{N} \cup\{\infty\})$ fails the ooKomlós property, but still has the oo-pre-Komlós property. We point out that the one-point compactification $\mathbb{N} \cup\{\infty\}$ of $\mathbb{N}$ is a separable compact metric space in which all points except $\infty$ are isolated.

It was mentioned in [5, Ex.5.3] that it was still unknown whether or not $C[0,1]$ has the nuo-pre-Komlós property. Here, we clarify the situation with Banach lattices $C(K)$ for a large class of compact Hausdorff spaces.

Theorem 2. Let $K$ be a compact Hausdorff space without isolated points in which there exist two distinct sequences $t_{n}$ and $t_{n}^{\prime}$ such that $\operatorname{cl}\left\{t_{n}\right\}_{n=1}^{\infty}=$ $c l\left\{t_{n}^{\prime}\right\}_{n=1}^{\infty}=K$. Then $C(K)$ fails the oo-pre-Komlós property.

Proof. Define $f_{k}(t)$ on $\left\{t_{n}\right\}_{n=1}^{k} \cup\left\{t_{n}^{\prime}\right\}_{n=1}^{k}$ to be equal to 1 if $t=t_{1}, \ldots, t_{k}$ and $f_{k}(t)=0$ if $t=t_{1}^{\prime}, \ldots, t_{k}^{\prime}$. Then extend each $f_{k}(t)$ continuously to whole $K$ so that $f_{k}(K) \subseteq[0,1]$. It is easy to see that, for any subsequence $f_{k_{j}}$ the sequence

$$
g_{m}=\frac{1}{m} \sum_{j=1}^{m} f_{k_{j}} \quad(m \in \mathbb{N})
$$

of Cesáro means is not oo-Cauchy.

Theorem 3. Let $K$ be a compact Hausdorff space with a nonempty separable open subset $U \subset K$ without isolated points such that every $u \in U$ has countable neighborhood base. Then $C(K)$ fails the oo-pre-Komlós property.

Proof. Let $D=\left\{d_{k}\right\}_{k=1}^{\infty}$ be a countable dense subset of a nonempty open subset $U \subset K$ without isolated points. Without lost of generality, we may choose countable neighborhood bases $B_{d}=\left\{U_{d}^{n}\right\}_{n=1}^{\infty}$ of elements $d \in D$ such that

$$
U_{d}^{n+1} \subseteq U_{d}^{n} \quad(\forall d \in D, n \in \mathbb{N})
$$

and $d_{m} \notin U_{d_{k}}^{k}$ for $m<k$.

We choose a sequence $\left\{d_{n_{k}}\right\}_{k=1}^{\infty}$ of distinct elements of $D$ as follows

$$
d_{n_{1}} \in U_{d_{1}}^{1}, d_{n_{2}} \in U_{d_{1}}^{2}, d_{n_{3}} \in U_{d_{2}}^{2}, d_{n_{4}} \in U_{d_{1}}^{3}, d_{n_{5}} \in U_{d_{2}}^{3}, d_{n_{6}} \in U_{d_{3}}^{3}, \ldots
$$


It is an easy exercise to show that $\operatorname{cl}\left\{d_{n_{2 k-1}}\right\}_{k=1}^{\infty}=\operatorname{cl}\left\{d_{n_{2 k}}\right\}_{k=1}^{\infty}=\operatorname{cl} U$.

By Theorem 2, there is a sequence $f_{k} \in \operatorname{cl} U$ such that $f_{k}(\operatorname{cl} U) \subseteq[0,1]$ for all $k$ with the property that for any subsequence $f_{k_{j}}$ the sequence

$$
g_{m}=\frac{1}{m} \sum_{j=1}^{m} f_{k_{j}} \quad(m \in \mathbb{N})
$$

of Cesáro means is not oo-Cauchy in $C(\operatorname{cl} U)$. Now, extend $f_{k}$ to $\bar{f}_{k} \in C(K)$ so that $\bar{f}_{k}(K) \subseteq[0,1]$ for all $k$. Clearly, for any subsequence $\bar{f}_{k_{j}}$ the sequence

$$
y_{m}=\frac{1}{m} \sum_{j=1}^{m} \bar{f}_{k_{j}} \quad(m \in \mathbb{N})
$$

is not oo-Cauchy in $C(K)$.

Corollary 2. For a compact metric space $K$ possessing a nonempty separable open subset without isolated points, the Banach lattice $C(K)$ fails the oo-pre-Komlós property.

Note that $\ell_{\infty} \cong C(\beta \mathbb{N})$ has oo-Komlós property (cf. e.g., [5, Ex.5.11]) From the other hand side, [5, Ex.5.2] implies that $\ell_{\infty}(\Gamma)$ fails the oo-preKomlós property, whenever $\operatorname{Card}(\Gamma) \geq c$.

\section{Komlós Sets}

The converse of the Komlós Theorem 1 has been proved in [8, Thm.2.1], namely Lennard has proved that: for a probability measure $\mu$, every convex $C \subset L_{1}(\mu)$ must be norm bounded provided that $C$ satisfies the property: for every sequence $x_{n}$ in $C$ there exist a subsequence $x_{n_{k}}$ and an $x \in E$, with

$$
x=u o-\lim _{m \rightarrow \infty} \frac{1}{m} \sum_{j=1}^{m} x_{n_{k_{j}}}
$$

for any subsequence $x_{n_{k_{j}}}$ of $x_{n_{k}}$. Subsets of any Banach lattice $E$ satisfying above property are called Komlós sets in [5, Def.5.22]. This motivates the following definition.

Definition 2. We say that $C \subset E$ is an $o^{-}$, uo-, $n$, or un-Komlós set (respectively, o-, uo-, $n$-, or un-pre-Komlós set) if, for every sequence $x_{n}$ in $C$, there exist a subsequence $x_{n_{k}}$ and $x \in E$ such that, for any further subsequence $x_{n_{k_{j}}}$, the sequence $g_{m}=\frac{1}{m} \sum_{j=1}^{m} x_{n_{k_{j}}}$ is o-, uo-, $n$-, or un-convergent to $x$ (respectively, $g_{m}$ is $o_{-}^{-}$, uo-, $n-$, or un-Cauchy). 
The main result of paper [5] concerning Komlós sets [5, Thm.5.23] can be read as follows.

Theorem 4 (Gao-Troitsky-Xanthos). Let $E$ be a Banach lattice with the projection property. If $E_{o c}$ is a norming subspace of $E^{*}$, then any convex uo-Komlós set $C$ in $E$ is norm bounded.

Below, in Proposition 4 we show that in arbitrary Banach lattice $E$ every convex uo-Komlós set $C \subseteq E_{+}$is norm bounded.

The following result shows that in [8, Thm.2.1] and in [5, Thm.5.23] uoKomlós sets can not be replaced by uo-pre-Komlós set.

Theorem 5. In any infinite dimensional Banach lattice $E$, there is an unbounded convex uo-pre-Komlós set $C \subseteq E_{+}$.

Proof. By [5, Cor.3.6, Cor.3.13], any disjoint sequence is a uo-Komlós set. Take a disjoint sequence $d_{n}$ in $E_{+}$such that $\left\|d_{n}\right\|=n$ for all $n$. Let $x_{i}=$ $\sum_{n=1}^{\infty} \alpha_{n}^{i} d_{n}$ be a sequence in the convex hull $C=\operatorname{co}\left\{d_{n}\right\}_{n=1}^{\infty}$. By diagonal argument, it is easy to find a subsequence $x_{i_{j}}$ satisfying

$$
\lim _{n \rightarrow \infty} \alpha_{n}^{i_{j}}=\beta_{n} \quad(\forall n) .
$$

By choosing further subsequence, if necessary, we may suppose that

$$
\left|\alpha_{n}^{i_{j}}-\beta_{n}\right| \leq 1 \quad(\forall j \geq n) .
$$

For $u=o-\sum_{n=1}^{\infty} n d_{n}, y=o-\sum_{n=1}^{\infty} \beta_{n} d_{n}$ in the universal completion $E^{u}$ (cf. [1, Def.7.20]) of $E$, by using (1) and (2) we get that

$$
\left|x_{i_{j}}-y\right| \leq \frac{1}{n} u
$$

for big enough $j$. In view of (3), the sequence $x_{i_{j}}$ o-converges to $y=o-$ $\sum_{n=1}^{\infty} \beta_{n} d_{n}$ in $E^{u}$, and hence is $u o$-Cauchy in $E$ by [5, 3.12]. It follows that every further subsequence $x_{i_{l}}$ is uo-Cauchy, and hence, by [5, 3.13], the sequence $g_{m}=\frac{1}{m} \sum_{l=1}^{m} x_{i_{l}}$ of its Cesáro means is uo-Cauchy as well.

Thus, $C$ is a norm unbounded convex uo-pre-Komlós set.

Proposition 4. In any Banach lattice $E$ every convex uo-Komlós set $C \subseteq$ $E_{+}$is norm bounded.

Proof. Let $C \subseteq E_{+}$be a norm unbounded convex set. We are going to show that $C$ is not uo-Komlós. 
Choose a sequence $x_{n} \in C$ so that $\left\|x_{1}\right\| \geq 4$ and

$$
\frac{1}{2^{n}}\left\|x_{n}\right\| \geq 2 \sum_{k=1}^{n-1} \frac{1}{2^{k}}\left\|x_{k}\right\| \quad(\forall n>1) .
$$

Define an increasing sequence $z_{n} \in E_{+}$as follows:

$$
z_{n}=\sum_{k=1}^{n} \frac{1}{2^{k}} x_{k}
$$

Clearly, every subsequence $z_{n_{k}}$ of $z_{n}$ is not uo-convergent, since otherwise $z_{n_{k}} \stackrel{\text { uo }}{\longrightarrow} z \in E_{+}$and $z-z_{n_{k}}=\left|z-z_{n_{k}}\right| \wedge z \stackrel{\mathrm{o}}{\rightarrow} 0$, which means that $z_{n_{k}} \uparrow z$, and hence $\left\|z_{n_{k}}\right\| \leq\|z\|<\infty$, violating

$$
\left\|z_{n}\right\| \geq \frac{1}{2^{n}}\left\|x_{n}\right\| \geq 2 \sum_{k=1}^{n-1} \frac{1}{2^{k}}\left\|x_{k}\right\| \geq 2\left\|z_{n-1}\right\| \geq 2^{n} \quad(\forall n \geq 1) .
$$

The sequence $w_{m}=\frac{1}{m} \sum_{j=1}^{m} z_{n_{k}}$ is also increasing and $\left\|w_{m}\right\| \rightarrow \infty$. The similar argument shows that $w_{m}$ is not uo-convergent. Let

$$
y_{n}=\frac{1}{2^{n}} x_{1}+z_{n} \in C \quad(\forall n \geq 1) .
$$

Since $\frac{1}{m} \sum_{k=1}^{m} \frac{1}{2^{n} k} x_{1} \leq \frac{1}{m} x_{1} \stackrel{\circ}{\rightarrow} 0$, the sequence of the Cesáro means

$$
\frac{1}{m} \sum_{k=1}^{m} y_{n_{k}}=\frac{1}{m} \sum_{k=1}^{m} \frac{1}{2^{n_{k}}} x_{1}+\frac{1}{m} \sum_{k=1}^{m} z_{n_{k}}=w_{m}+\frac{1}{m} \sum_{k=1}^{m} \frac{1}{2^{n_{k}}} x_{1} \quad(m \in \mathbb{N})
$$

is not uo-convergent. It shows that $C$ is not $u o$-Komlós.

Note that the similar argument, as in the proof of Proposition 4, shows that in any Banach lattice $E$ every convex un-Komlós set $C \subseteq E_{+}$is norm bounded.

The next result follows directly from Theorem 5 and Proposition 4.

Theorem 6. Let $E$ be an infinite dimensional Banach lattice. Then there exists a norm unbounded convex uo-pre-Komlós set $C \in E_{+}$which is not uo-Komlós.

Corollary 3. Let $E$ be an Banach lattice. The following conditions are equivalent:

(1) $\operatorname{dim}(E)<\infty$;

(2) $E$ is uo-complete;

(3) E is sequentially uo-complete;

(4) every uo-pre-Komlós set is uo-Komlós; 
(5) every convex uo-pre-Komlós set $C \in E_{+}$is norm bounded.

Proof. Implications (1) $\Rightarrow(2) \Rightarrow(3)$ are trivial, and $(3) \Rightarrow(4)$ easily follows from Definition 2 ,

$(4) \Rightarrow(5)$ : Let $C \in E_{+}$be a convex uo-pre-Komlós set. Then $C$ is uoKomlós by the assumption. Proposition 4 ensures that $C$ is norm bounded.

$(5) \Rightarrow(1)$ : It is exactly Theorem 5 .

\section{REFERENCES}

[1] C. D. Aliprantis and O. Burkinshaw, Locally solid Riesz spaces with applications to economics, second ed., Mathematical Surveys and Monographs, Vol. 105, American Mathematical Society, Providence, RI, 2003.

[2] C. D. Aliprantis and O. Burkinshaw, Positive operators, Springer, Dordrecht, 2006, Reprint of the 1985 original.

[3] Y. A. Dabboorasad, E. Y. Emelyanov, and M. A. A. Marabeh, umTopology in multi-normed vector lattices, Positivity (to appear).

[4] Y. Deng, M. O'Brien, and V. G. Troitsky, Unbounded Norm Convergence in Banach Lattices, Positivity 21 (2017), 963-974.

[5] N. Gao, V. G. Troitsky, and F. Xanthos, Uo-convergence and its applications to Cesáro means in Banach lattices, Israel Journal of Math. 220 (2017), 649-689.

[6] M. Kandić, M. A. A. Marabeh, and V. G. Troitsky, Unbounded norm topology in Banach lattices, J. Math. Anal. Appl. 451 (2017), 259279.

[7] J. Komlós, A generalization of a problem of Steinhaus, Acta Math. Acad. Sci. Hungar. 18 (1967), 217-229.

[8] C. Lennard, A converse to a theorem of Komlós for convex subsets of $L_{1}$, Pacific J. Math. 159 (1993), 75-85.

1 Department of Mathematics, Middle East Technical University, 06800 ANKARA, TURKEY.

${ }^{2}$ Department of Mathematics, Hacettepe University, 06800 Ankara, Turkey.

3 Sobolev Institute of Mathematics, 630090 Novosibirsk, Russia.

E-mail address: eduard@metu.edu.tr, erkursun.ozcan@hacettepe.edu.tr, lanagor71@gmail.com 